\title{
Polarization-interference images of optically anisotropic biological layers
}

M. Yu. Sakhnovskiy, O. Yu. Wanchuliak, B. Bodnar, I. V. Martseniak, O. Tsyhykalo, et al.

M. Yu. Sakhnovskiy, O. Yu. Wanchuliak, B. Bodnar, I. V. Martseniak, O. Tsyhykalo, A. V. Dubolazov, V. A. Ushenko, O. I. Olar, P. M. Grygoryshyn, "Polarization-interference images of optically anisotropic biological layers," Proc. SPIE 10752, Applications of Digital Image Processing XLI, 107522F (17 September 2018); doi: 10.1117/12.2320509

Event: SPIE Optical Engineering + Applications, 2018, San Diego, California, United States 


\title{
Polarization-interference images of optically anisotropic biological layers
}

\author{
M. Yu. Sakhnovskiy ${ }^{1}$, O.Yu.Wanchuliak ${ }^{2}$, B.Bodnar ${ }^{2}$,I.V.Martseniak ${ }^{1}$, O.Tsyhykalo², A.V. \\ Dubolazov $^{1 *}$, V.A. Ushenko ${ }^{1}$, O.I.Olar ${ }^{2}$, P.M. Grygoryshyn ${ }^{2}$ \\ ${ }^{1}$ Chernivtsi National University, 2 Kotsiubynskyi Str., Chernivtsi, Ukraine, 58012 \\ ${ }^{2}$ Bukovinian State Medical University, 3 Theatral Sq., Chernivtsi, Ukraine, 58000
}

a.dubolazov@,chnu.edu.ua

\begin{abstract}
A theoretical basis for the method of polarization-interference mapping of optically thin polycrystalline films of human biological fluids is given. The coordinate distributions of the value of the local contrast of the interference distributions of the polarization-inhomogeneous microscopic images of polycrystalline films of the synovial fluid of the human joint are investigated. In the framework of the statistical (statistical moments of the 1st-4th order) approaches, objective criteria for the distribution of the values of local contrast are established. The possibility of differentiation of weak changes in the optical anisotropy of blood films of healthy and patients with breast cancer patients is determined.
\end{abstract}

Keywords: polarization, interference, anisotropy, cartography.

\section{Introduction}

Among the many areas of optical diagnostics of biological objects, the original place is occupied by laser polarimetry of optically thin (nondepolarizing) layers of biological tissues ${ }^{1-15}$. The main object of this diagnosis is a variety of tumor or precancerous conditions of tissues of human organs with fibrillar structure of polycrystalline networks. In ${ }^{16-21}$, a high sensitivity of the polarimetric differentiation of such samples was demonstrated. It has been established that such pathologies are accompanied by significant changes in phase anisotropy due to the transformation of the optical axes direction distributions and the birefringence of protein fibrils ${ }^{22-27}$. At the same time, obtaining samples of histological sections requires a traumatic biopsy operation. This circumstance complicates the application of laser polarimetry methods of biological tissues in everyday clinical practice. To overcome this disadvantage, it is promising to study the distributions of the polarization-phase parameters of microscopic images of smears of biological fluids. Such objects are easily accessible, which makes it possible to carry out a non-traumatic evaluation of the presence of a pathology at an early stage of its occurrence. This circumstance opens up new possibilities for using methods of polarimetry in screening mass surveys.

In our work we consider the polarization-interference mapping of such layers. The purpose of such studies is to reveal the interrelationships between the local contrast divisions of interference patterns and phases in the plane of images of polycrystalline films and the changes in their birefringence. An applied aspect of the work is the determination of the effectiveness of differentiation of polycrystalline blood films of healthy and patients with breast cancer patients.

\section{A brief theory of polarization-interference mapping of polycrystalline films}

Let us consider the process of forming an interference distribution in the plane of an interference-inhomogeneous image of an optically thin (nondepolarizing) polycrystalline film of a biological fluid. We use the following approximations:

- $\quad$ irradiating and reference waves are circularly polarized $\left\{\begin{array}{l}\Phi_{y}-\Phi_{x}=0.5 \pi ; \\ \varphi_{y}-\varphi_{x} \equiv 0.5 \pi ;\end{array} ;\left\{\begin{array}{l}\left|B_{x}\right|=\left|W_{x}\right| \equiv 1 ; \\ \left|B_{y}\right|=\left|w_{y}\right| \equiv 1 ;\end{array}\right.\right.$

Applications of Digital Image Processing XLI, edited by Andrew G. Tescher, Proc. of SPIE Vol.

10752, 107522F - (c) 2018 SPIE · CCC code: 0277-786X/18/\$18 - doi: 10.1117/12.2320509 


$$
B=W=\left(\begin{array}{l}
1 \\
i
\end{array}\right)
$$

- phase fluctuations of the polycrystalline layer are small

$$
\begin{gathered}
\vartheta \rightarrow 0: \cos \vartheta \approx 1 ; \sin \vartheta \approx \vartheta \\
\left\{\begin{array}{l}
m_{11}=1-i \vartheta \sin ^{2} \vartheta \\
m_{12}=d_{21}=i \vartheta \cos \alpha \sin \alpha \\
m_{22}=1-i \cos ^{2} \alpha
\end{array}\right.
\end{gathered}
$$

It is shown that the intensity of the interference field at the point $r$

$$
D(a)=\vartheta^{2}(a)-2 \vartheta(a) \sin \beta(a)+2 \cos \beta(a) .
$$

From analysis (5) it follows that

$$
\left\{\begin{array}{l}
\beta(a)=0 \leftrightarrow D_{\max }(a)=2+\vartheta^{2}(a) \\
\beta(a)=\pi \leftrightarrow I_{\min }(a)=\vartheta^{2}(a)
\end{array}\right.
$$

The local contrast of the interference distribution is given by

$$
V(\beta, \vartheta)=\frac{D_{\max }^{(\beta=0)}-D_{\min }^{(\beta=\pi)}}{D_{\max }^{(\beta=0)}+D_{\min }^{(\beta=\pi)}}=\frac{1}{1+\vartheta^{2}} .
$$

Thus, by means of circular polarization-interference mapping of a microscopic image of a polycrystalline biological fluid, it is possible to obtain an azimuthally invariant distribution (within the pixels of a digital camera) of the magnitude of the local contrast $V(m \times n)=W\left(\begin{array}{ccc}a_{11} & \ldots & a_{1 n} \\ . & \ldots & . \\ a_{m 1} & \ldots & a_{m n}\end{array}\right)$ and to estimate the weak changes in the birefringence of this layer.

\section{Materials and methods}

In fig. 1 shows the optical scheme of polarization interferometry of laser microscopic images of polycrystalline films of biological fluids. 


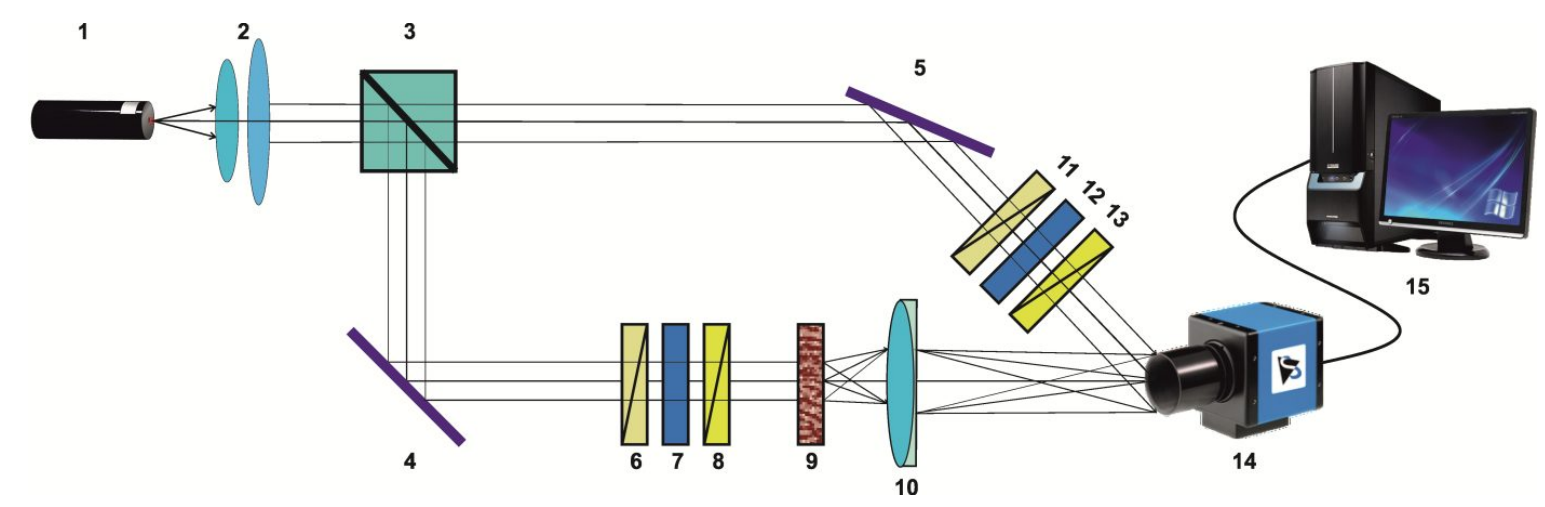

Fig. 1. Optical scheme of polarization interferometry of microscopic images of optically anisotropic biological layers. Explanations in the text

The parallel $\left(\varnothing=2 \times 10^{3} \mu \mathrm{m}\right)$ beam He-Ne $(\lambda=0,6328 \mu \mathrm{m})$ of laser 1 , formed with the help of a collimator 2 , the beam splitter 3 is divided into two - "irradiating" and "reference".

The "irradiating" beam with the help of the rotary mirror 4 is guided through the polarization filter $6-8$ in the direction of the sample of the biological layer 9. The polarization-inhomogeneous image of the object 9 by the objective 10 (Nikon CFI Achromat P, focal length $30 \mathrm{~mm}$, numerical aperture 0.1, magnification - 4x) is projected into the plane of the photosensitive pad $(m \times n=1280 \times 960$ pixels $)$ of the digital camera 14 .

The "reference" beam by the mirror 5 is guided through the polarization filter 11-13 into the plane of the polarizationinhomogeneous image of the object 9. As a result, an interference pattern is formed, the coordinate intensity distribution is recorded by the digital camera 14 (The Imaging Source DMK 41AU02.AS, monochrome 1/2 "CCD, Sony ICX205AL (progressive scan); the resolution is $1280 \times 960$; the size of the light-sensitive pad is $7600 \times 6200 \mu \mathrm{m}$; the sensitivity is 0,05 $\mathrm{lx}$; the dynamic range is 8 bit; SNR is 9 bit).

\subsection{Method for measuring the coordinate distributions of the local contrast value of interference patterns of polarization-inhomogeneous images}

- $\quad$ polarizers 8 and 13 "are output" from the path of the irradiating beam (Fig. 1);

- the axes of the highest velocity of quarter-wave plates 7 and 12 are oriented at an angle $\Omega=45^{0}$ with respect to the transmission axis of the polarizers 6 and 11;

- the digital camera 14 registers a discrete $(m \times n)$ image of the interference intensity distribution in the plane of the microscopic image of the polycrystalline film;

- the registered distribution $I(m \times n)$ is scanned by the aggregate $(k=1, \ldots m)$ of the rows $(k 1, \ldots k n)$ in increments $\Delta r=1 p i x$;

- for every k-th row, a set of local contrast values is determined from the following relationship

$$
V_{k}=\frac{\left|D\left(a_{i=1, \ldots n}\right)-D\left(a_{i=1, \ldots n}+\Delta a\right)\right|}{D\left(a_{i=1, \ldots n}\right)+D\left(a_{i=1, \ldots n}+\Delta a\right)}
$$

- for the whole set of series, a coordinate distribution (map) of the value of the local contrast of the interference distribution in the plane of the microscopic image of a polycrystalline biological film 


$$
V(m \times n)=\left(\begin{array}{ccc}
V_{11} & \ldots & V_{1 n} \\
\cdot & \ldots & \cdot \\
V_{m 1} & \ldots & V_{m n}
\end{array}\right)
$$

\section{Brief description of the objects of research.}

The following optically thin (attenuation coefficient $\tau \prec 0.01$ ) samples of polycrystalline films (geometric thickness $l=15 \mu m 0.0089 \leq \tau \leq 0.0097$ ) of blood were studied:

- $\quad \operatorname{norm}(25$ samples), - group 1;

- breast cancer (25 samples), - group 2.

In Fig. 2 presents microscopic images of polycrystalline group 1 (fragment (1)) and group 2 (fragment (2)).

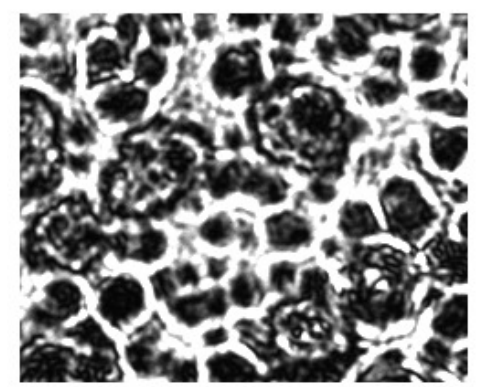

(1)

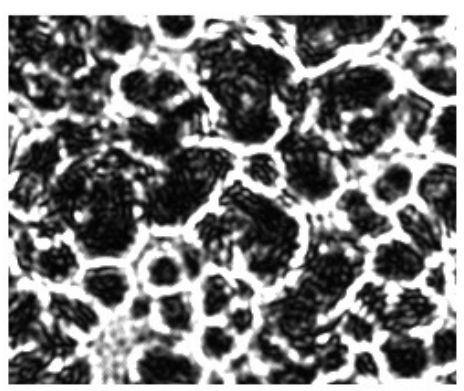

(2)

Fig. 2. Microscopic images of polycrystalline blood films healthy (fragment (1)) and patients with breast cancer (fragment (2)) of patients.

A comparative analysis of the reduced microscopic images revealed a polycrystalline structure of the blood films similar in orientation to the structure. The main optically anisotropic components of such a liquid are birefringent albumin, globulin and uniform elements. Due to linear birefringence, a coordinate distribution of the phase shifts between the linearly polarized orthogonal components of the laser radiation amplitude is formed.

\section{Experimental results and their discussion}

In fig. 3. polarization-interference images of polycrystalline blood films of healthy (fragment (1)) and a patient with cancer of the blood (fragment (2)) of patients are presented.

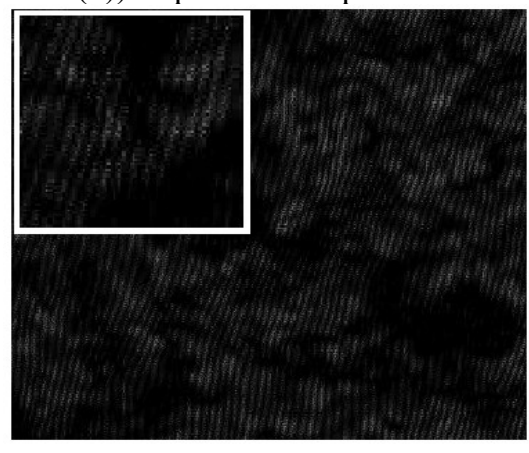

(1)

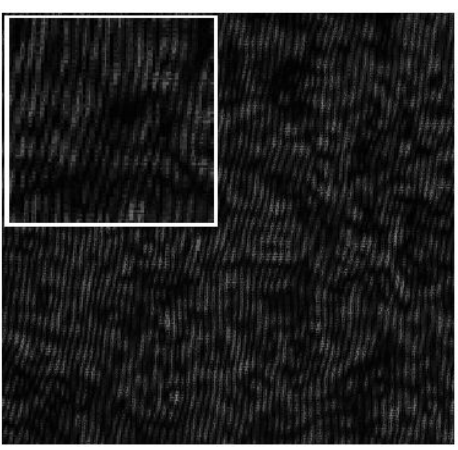

(2)

Fig. 3. Polarization-interference images of polycrystalline blood films of patients from group 1 (fragment (1)) and group 2 (fragment (2)). 
In fig. 4 presents the results of the determination of the coordinate distributions of the local contrast value of polycrystalline blood films of healthy (fragments (1), (3)) and patients with breast cancer (fragment (2), (4)) of patients.

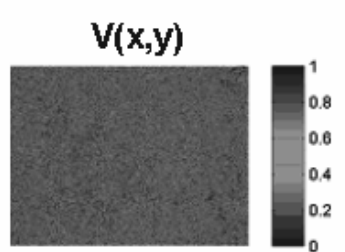

(1)

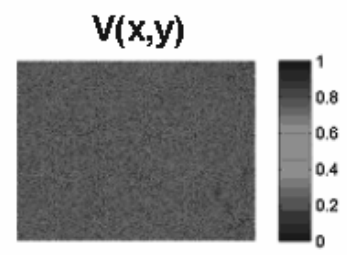

(3)

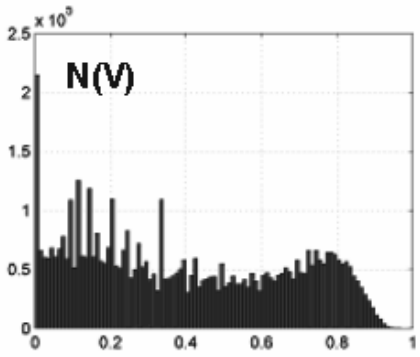

(2)

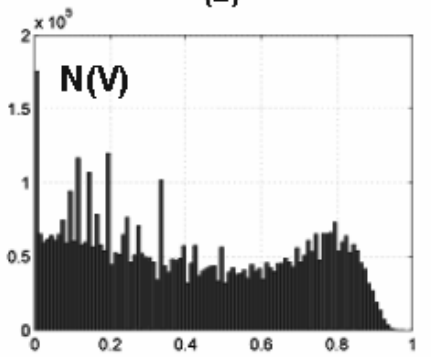

(4)

Fig. 4. Maps and histograms of the distribution of local contrast values of polarization-interference distributions in the plane of images of polycrystalline blood films of healthy (fragments (1), (3)) and patients with breast cancer (fragment (2), (4)) of patients.

A comparative analysis of the obtained maps and histograms of distributions of local contrast values of polarizationinterference patterns of polycrystalline films of blood of both groups of patients revealed:

- practically the maximum range of changes in the value of local contrast $0 \leq V \leq 0.9$ (fig. 4 , fragments (2), (4));

- histograms $N(V)$ of distributions of random values of local contrast $V$ for both interference patterns have a complex asymmetric structure with the presence of a series of individual extrema that are in different parts of the interval of change $V-N_{\max }(V) \approx 0 ; \quad 0,2 \leq N_{\max }(V) \leq 0,35 ; \quad N_{\max }(V) \approx 0,8$ (Fig. 4, fragments (2), (4));

- for the histogram $N(V)$ calculated for the polarization-interference distribution in the plane of the microscopic image of the polycrystalline film of blood from group 2, there is a high probability of high local contrast values - the extremes of the minimum and maximum values $V$ vary according to the scenario $-N_{\max }(V) \approx 0 \downarrow$ and $N_{\text {max }}(V) \approx 0,8 \uparrow$ (Fig. 4, fragment (4)).

Table 1 shows the average values and mean errors of statistical $\left(Z_{i=1 ; 2 ; 3 ; 4}\right)$, which characterize the distributions of the local contrast $W$ value of polarization-interference distributions (Fig. 4, fragments (2), (4)) of images of polycrystalline blood films of both groups of patients.

Table 1 Statistical parameters of local contrast distributions of polycrystalline blood films

\begin{tabular}{|c|c|c|c|}
\hline Samples & Norm & Cancer & $A c(W), \%$ \\
\hline$Z_{1}$ & $0,092 \pm 0,0049$ & $0,13 \pm 0,0074$ & 86 \\
\hline$Z_{2}$ & $0,14 \pm 0,074$ & $0,19 \pm 0,0089$ & 77 \\
\hline$Z_{3}$ & $1,36 \pm 0,081$ & $2,35 \pm 0,14$ & 92 \\
\hline \hline$Z_{4}$ & $0,87 \pm 0,051$ & $0,51 \pm 0,029$ & 91 \\
\hline
\end{tabular}


The conducted cycle of studies of the polarization-interference structure of microscopic images of polycrystalline films of blood was found to be excellent ( $90 \% \leq A c(W) \leq 92 \%)$ the quality of the diagnostic test $\left.{ }^{28-30}\right)$.

\section{Conclusion}

A new method of polarization-interference mapping of polarization-inhomogeneous images of polycrystalline films of biological fluids with objective statistical analysis of local contrast maps is proposed and analytically justified.

Objective criteria characterizing maps of local contrast of interference distributions of images of polycrystalline blood films of healthy and patients with breast cancer patients are determined.

An analysis of the results of the diagnostic efficiency of the method of polarization-interference mapping of microscopic images of the investigated samples of polycrystalline films demonstrated an excellent $(A c \succ 90 \%)$ accuracy of differential diagnostics of changes in the optical anisotropy of blood films.

\section{References}

[1]. Wang X. Propagation of polarized light in birefringent turbid media: a Monte Carlo study / X. Wang, L.-H. Wang // J. Biomed. Opt. - 2002. - Vol. 7. - P. 279-290.

[2]. Tuchin V.V.Handbook of optical biomedical diagnostics/V.V. Tuchin.-Bellingham: SPIE Press, 2002. - 1110p.

[3]. Yao G. Two-dimensional depth-resolved Mueller matrix characterization of biological tissue by optical coherence tomography / G. Yao, L. V. Wang // Opt. Lett. - 1999.- V.24.-P. 537-539.

[4]. Tower T. T. Alignment Maps of Tissues: I. Microscopic Elliptical Polarimetry / T. T. Tower, R. T. Tranquillo // Biophys. J. - 2001. - Vol. 81. - P. 2954 2963.

[5]. Lu S. Interpretation of Mueller matrices based on polar decomposition/S. Lu, R. A. Chipman// J. Opt. Soc. Am. A. -1996. - Vol. 13. -P.1106-1113.

[6]. Ghosh Nirmalya. Techniques for fast and sensitive measurements of two-dimensional birefringence distributions / Nirmalya Ghosh, I. Alex Vitkin // Journal of Biomedical Optics.-2011.-№ 16(11).-P. 110801.

[7]. V. V. Tuchin, L. Wang, and D. A' . Zimnyakov, Optical Polarization in Biomedical Applications, New York, USA(2006).

[8]. Angelsky, O.V., Maksimyak, A.P., Maksimyak, P.P., Hanson, S.G., "Optical correlation diagnostics of rough surfaces with large surface inhomogeneities," Optics Express, 14 (16), 7299-7311(2006).

[9]. Ushenko, A.G., "The vector structure of laser biospeckle fields and polarization diagnostics of collagen skin structures," Laser Physics, 10 (5), 1143-1149 (2000).

[10]. Ushenko, Alexander G., "Polarization structure of laser scattering field," Optical Engineering, 34 (4), $1088-1093$ (1995).

[11]. Angelsky, O.V., Maksimyak, P.P., Ryukhtin, V.V., Hanson, S.G., "New feasibilities for characterizing rough surfaces by optical-correlation techniques," Applied Optics, 40 (31), 5693-5707 (2001).

[12]. Angelsky, O.V., Ushenko, A.G., Ushenko, Ye.G., "Investigation of the correlation structure of biological tissue polarization images during the diagnostics of their oncological changes," Physics in Medicine and Biology, 50 (20), 4811-4822 (2005).

[13]. Angel'skiǔ, O.V., Ushenko, A.G., Arkhelyuk, A.D., Ermolenko, S.B., Burkovets, D.N., "Scattering of laser radiation by multifractal biological structures," Optics and Spectroscopy (English translation of Optika i Spektroskopiya), 88 (3), $444-447$ (2000).

[14]. Angelsky, O.V., Gorsky, M.P., Hanson, S.G., Lukin, V.P., Mokhun, I.I., Polyanskii, P.V., Ryabiy, P.A., “Optical correlation algorithm for reconstructing phase skeleton of complex optical fields for solving the phase problem,” Optics Express, 22 (5), pp. 6186-6193 (2014).

[15]. Angelsky, O.V., Bekshaev, A.Ya., Maksimyak, P.P., Maksimyak, A.P., Hanson, S.G., "Measurement of small light absorption in microparticles by means of optically induced rotation," Optics Express, 23 (6), 7152-7163 (2015).

[16]. Ushenko, V.A., Koval, G.D., Gavrylyak, M.S., "Mueller - Matrices polarization selection of two-dimensional linear and circular birefringence images," Proceedings of SPIE - The International Society for Optical Engineering 8856, 88562E (2013)

[17]. Prysyazhnyuk, V.P., Ushenko, Yu.A., Dubolazov, A.V., Ushenko, A.G., Ushenko, V.A., "Polarization-dependent laser autofluorescence of the polycrystalline networks of blood plasma films in the task of liver pathology differentiation," Applied Optics 55 (12), B126-B132 (2016)

[18]. Ushenko, V.A., "Complex degree of mutual coherence of biological liquids," Proceedings of SPIE - The International Society for Optical Engineering 8882, 88820V (2013) 
[19]. Ushenko, Y.A., Koval, G.D., Ushenko, A.G., Dubolazov, O.V., Ushenko, V.A., Novakovskaia, O.Y., "Mueller-matrix of laserinduced autofluorescence of polycrystalline films of dried peritoneal fluid in diagnostics of endometriosis," Journal of Biomedical Optics 21 (7), 071116 (2016)

[20]. Ushenko, V.O., "Spatial-frequency polarization phasometry of biological polycrystalline networks," Optical Memory and Neural Networks (Information Optics) 22 (1), 56-64(2013)

[21]. Angelsky, P.O., Ushenko, A.G., Dubolazov, A.V., Sidor, M.I., Bodnar, G.B., Koval, G., Trifonyuk, L., "The singular approach for processing polarization-inhomogeneous laser images of blood plasma layers," Journal of Optics (United Kingdom) 15 (4), $044030(2013)$

[22]. Ushenko, O.G., Dubolazov, A.V., Balanets'ka, V.O., Karachevtsev, A.V., Sydor, M., "Wavelet analysis for polarization inhomogeneous laser images of blood plasma," Proceedings of SPIE - The International Society for Optical Engineering 8338, $83381 \mathrm{H}(2011)$

[23]. Angelsky, O.V., Ushenko, Y.A., Dubolazov, A.V., Telenha, O.Yu., "The interconnection between the coordinate distribution of Mueller-matrixes images characteristic values of biological liquid crystals net and the pathological changes of human tissues," Advances in Optical Technologies, 130659 (2010)

[24]. Dubolazov, A.V., Marchuk, V., Olar, O.I., Bachinskiy, V.T., Vanchuliak, O.Ya., Pashkovska, N.V., Andriychuk, D., Kostiuk, S.V., "Multiparameter correlation microscopy of biological fluids polycrystalline networks," Proceedings of SPIE - The International Society for Optical Engineering 9066, 90661Y (2013)

[25]. Ushenko, Y.O., Tomka, Y.Y., Dubolazov, O.V., Balanetska, V.O., Karachevtsev, A.V., Angelsky, A.-P., "Wavelet-analysis for laser images of blood plasma," Advances in Electrical and Computer Engineering 11 (2), pp. 55-62(2011).

[26]. Ushenko, V.A., Dubolazov, A.V., "Correlation and self similarity structure of polycrystalline network biological layers mueller matrices images," Proceedings of SPIE - The International Society for Optical Engineering 8856, 88562D (2013).

[27]. Prysyazhnyuk, V.P., Ushenko, Yu.A., Dubolazov, A.V., Ushenko, A.G., Ushenko, V.A., "Polarization-dependent laser autofluorescence of the polycrystalline networks of blood plasma films in the task of liver pathology differentiation," Applied Optics 55 (12), B126-B132(2016).

[28]. "Cassidy, "Basic concepts of statistical analysis for surgical research," Journal of Surgical Research 128,199-206 (2005).

[29]. C. S. Davis, Statistical methods of the analysis of repeated measurements, 744, New York: Springer-Verlag (2002).

[30]. A. Petrie, B. Sabin, Medical Statistics at a Glance, pp. 157, Blackwell Publishing (2005). 